\title{
Photonic crystal waveguides in artificial opals
}

Lavrinenko, Andrei; Kiyan, Roman; Neumeister, Andrei; Schweizer, Thorsten; Leyrer, Reinhold J.; Wohlleben, Wendel

\section{Published in:}

10th Anniversary international Conference on Transparent Optical Networks

Link to article, DOI:

10.1109/ICTON.2008.4598593

Publication date:

2008

Document Version

Publisher's PDF, also known as Version of record

Link back to DTU Orbit

Citation (APA):

Lavrinenko, A., Kiyan, R., Neumeister, A., Schweizer, T., Leyrer, R. J., \& Wohlleben, W. (2008). Photonic crystal waveguides in artificial opals. In 10th Anniversary international Conference on Transparent Optical Networks (Vol. 2, pp. 64-67). IEEE. https://doi.org/10.1109/ICTON.2008.4598593

\section{General rights}

Copyright and moral rights for the publications made accessible in the public portal are retained by the authors and/or other copyright owners and it is a condition of accessing publications that users recognise and abide by the legal requirements associated with these rights.

- Users may download and print one copy of any publication from the public portal for the purpose of private study or research.

- You may not further distribute the material or use it for any profit-making activity or commercial gain

- You may freely distribute the URL identifying the publication in the public portal 


\title{
Photonic Crystal Waveguides in Artificial Opals
}

\author{
Andrei V. Lavrinenko ${ }^{1}$, Roman Kiyan ${ }^{2}$, André Neumeister ${ }^{2}$, Thorsten Schweizer ${ }^{2}$ \\ Reinhold J. Leyrer ${ }^{3}$, Wendel Wohlleben ${ }^{3}$ \\ ${ }^{1}$ DTU Fotonik, Department of Photonics Engineering, NanoDTU, Technical University of Denmark \\ Building 345V, DK-2800, Kgs. Lyngby, Denmark \\ ${ }^{2}$ Laser Zentrum Hannover e.V., Hollerithallee 8, 30419 Hannover, Germany \\ ${ }^{3}$ Polymer Research, BASF Aktiengesellschaft, 67056 Ludwigshafen, Germany \\ Tel: (45) 4525 6392,Fax: (45) 4593 6581,e-mail: ala@com.dtu.dk
}

\begin{abstract}
3D photonic crystals based on Si inverted-opals are numerically explored as hosts for effective air-channel waveguides, which can serve as parts of photonic circuits. Two basic shapes of straight waveguides are considered: cylindrical and a chain of spheres. Modelling shows that transmission is heavily dependent on the lattice position of the waveguide and its direction. Our experiments of defect inscription by 2-photon polymerization for the production of straight and bent waveguides in opal templates are reported.

Keywords: three-dimensional photonic crystals, photonic crystal waveguides, FDTD, two-photon polymerization, imperfections.
\end{abstract}

\section{INTRODUCTION}

To fully exploit the advantages of the photonic crystal waveguide (PCW) concept in communications, the production of 3D photonic circuits is necessary. We explore possibilities to employ artificial opals as a technological platform for the defect inscription. Fabrication of polymer based millimetre-scale colloidal opals free from the macro defects is the first target. Polydispersity of spheres should be within the tolerance frames revealed in numerical experiments of imperfections influences on the photonic properties of opals. The next crucial step in achieving the target is inscribing arbitrarily shaped, sized and oriented waveguides in the bulk $3 \mathrm{D}$ photonic crystals. The inscription of defined defects by two-photon polymerization (2PP) of a photo polymer infiltrated in 3D photonic band gap templates has been identified as a possible technique to fabricate 3D photonic components and circuits. Using the 2PP technique, a liquid polymer is transformed into a solid threedimensional object by freely moving focused laser pulses in the femtosecond (fs) regime inside the liquid material. Absorption of energy by the material only takes part in the focal volume, thus curing of the liquid can be achieved below the photopolymer surface, allowing for true 3D structuring. The resulting spatial features can be less than a cubic micron in volume and one-dimensional characteristics features as small as $100 \mathrm{~nm}$ [1], which is sufficient for components operating in the infrared telecommunication windows. Multimode waveguides and cavities fabricated by the 2PP technique have been reported recently [2]. Prior to the development of production techniques, careful design and simulation of the templates and photonic crystals should be performed. We report on the quantitative study of disorder influence on light transmission in threedimensional photonic crystals having a complete photonic band gap like Si-inverted opals. Disorder is implied as fluctuation in sizes and/or sites of air spheres. Two kinds of disorder distributions are used in modelling: Gaussian and a skew one obtained from checking colloidal particles distribution before the segmentation in polystyrene opal template. The results of the simulations of disorder influence are reported in Section 2. The design of the PCWs is described in Section 3. Section 4 contains some examples of PCWs fabricated by the 2PP technique.

\section{FABRICATIONAL TOLERANCES FOR SI INVERTED OPALS AS A PERFECT PHOTONIC INSULATOR}

A 3D photonic crystal possessing a complete photonic band gap (PBG) is actually nothing else but a matrix providing perfect photonic insulation for the integrated circuits incorporated inside. It is important for manufacturing purposes to analyse the resistance of the Si inverted opal platform with respect to possible errors in arrangement of elements of its spatial lattice. There are several sources of structural imperfections: variations in positions, changes in radius and shape, surface roughness, and doping of various substances. Some analysis of the influence of position and radius imperfections on the opening of the complete PBG in inverted opals was done in the literature, however with the uniform statistical distribution only [3], which cannot give sufficient feedback to the fabrication chain.

We attempt to make such analysis with the Gaussian distribution of radii and sites of polymer spheres by utilizing transmission characteristics of thin opal films [4]. In addition to the normally distributed deviations we utilized statistical polydispersity distribution measured in solution with the analytical ultracentrifugation method

This work is supported by the European Commission FP6, project NewTon, NMP4-CT-2005-017160. 
[4] providing high resolution. The actual distribution of polymer dispersions is not symmetrical but skewed towards larger spheres. We will refer hereafter to this distribution as D1. The best normal fit to the D1 has mean standard deviation $\sigma \approx 0.02$ (Fig. 1, left). Radius imperfections with mean standard deviation $\sigma=0.04$ diminish the complete PBG. Similar results occur with deviations of positions of spheres from the FCC arrangement. Significant shrinkage of the PBG sizes is observable for imperfections in sizes and sites distributed normally with $\sigma=0.02$. Calculations on infinite periodic crystals show closing of band gaps with $\sigma=0.04$ imperfections in site and size [3]. Our results put stricter constraints on the fabrication quality for real, e.g. finite systems.
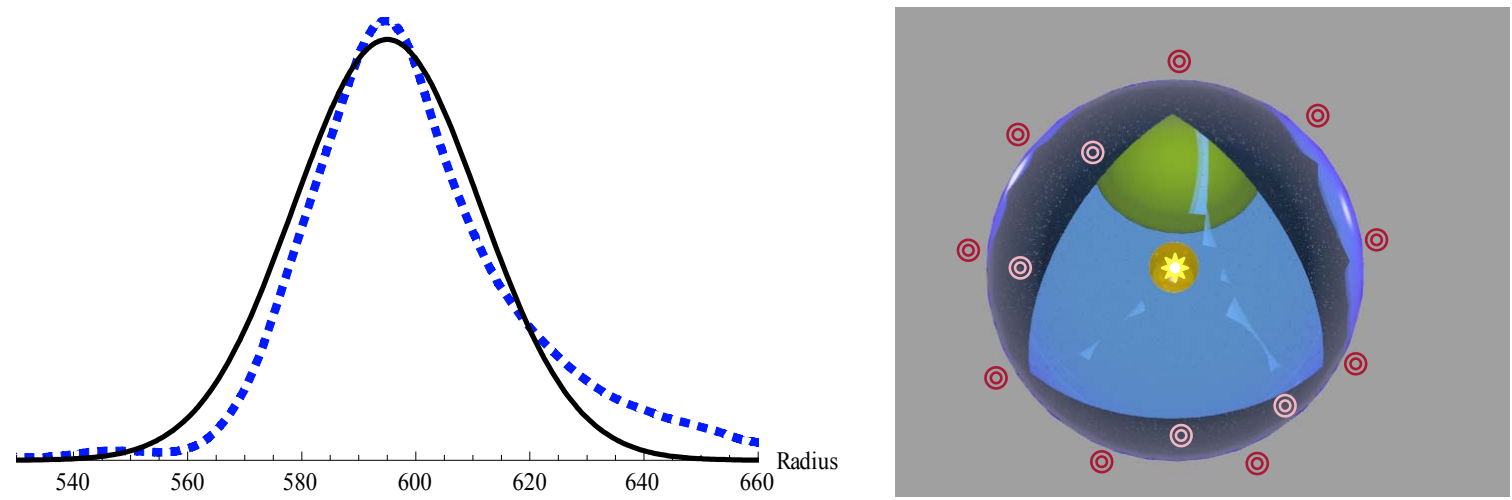

Figure 1. Left: the best Gaussian fit (solid curve) with $\sigma=0.02$ to the skew distribution (dashed curve); Right: the layout of the inverted opal sphere used for tests on photonic insulation. The point-like source is in the centre, detectors (black spots) are placed on the same distances from the source around the sphere.

Here we focus also on another approach attempting to answer the question: how large can be imperfections providing still essential photonic insulation by inverted opal. We take a big sphere made of Si inverted opal. Diameter of the sphere is $10 a$, where $a$ is a lattice constant. In the centre of the sphere we insert a point source consisting of two dipoles of mutually orthogonal polarizations (Fig. 1, right). A set of point detectors is placed at the same distance from the centre (one grid point away from the surface), but at different directions seen from the centre. There are three options: (i) 1000 randomly placed detectors; (ii) 184 detectors homogeneously covering the surface and (iii) detectors arranged in the principle highly symmetrical directions of the FCC lattice: 6 in $\Gamma$-X, 8 in $\Gamma$-L, 12 in $\Gamma-\mathrm{K}, 24$ in $\Gamma$-W. After FDTD-run completion and processing information about fields stored by the detectors (transforming into the frequency domain and averaging on one detector from each group of detectors) we obtain data, which tell us how large the fields leaking from the sphere in specific directions connected to the crystal symmetry or in all directions in average are. Typically we plot the intensity of the fields sensed by the detectors. Such characteristics are similar to the well known density of optical states calculations (DOS), this is why we refer to them as quasi-DOS (QDOS). An analysis of the imperfections influence on the closing of the PBG in specific directions can be made with the help of plots shown in Fig. 2.

The critical values of imperfections which lead to the closing of the PBG are: (i) Imperfections in positions of spheres with $\sigma \geq 0.04$; (ii) imperfections in radius with $\sigma \geq 0.03$, (iii) influence is stronger than in the sitecase, also mentioned in [3]; (iv) imperfections in both radii and positions (s\&s) with $\sigma \geq 0.02$. Imperfections obeyed the skew (D1) statistical distribution have much worse influence on the PBG properties than the normally distributed $\mathrm{s} \& \mathrm{~s}$ imperfections with $\sigma=0.02$ (the best normal fit). Thus we postulate the ultimate frames for the process of opal fabrication. However, the strongest concern is what happens during the inversion process, where high temperature conditions critically affected the quality of the opal template.

\section{DESIGN OF WAVEGUIDES IN SI INVERTED OPALS}

The main goal in designing of photonic components is to investigate possibility of effective transportation of light in waveguides inserted as straight defects in the $\mathrm{Si}$ inverted opal platform taken as photonic insulators. Following [5] we consider two principle shapes of PCWs. Cylindrical photonic crystal waveguides (CPCW) are made as a straight cylindrical air defects inserted in an inverted opal structure. Another waveguide design is a chain of spheres (CoS), arranged with the same spatial periodicity as the structural elements of the crystal lattice. To simplify our task we use three principal orientations of the symmetric planes: (i) direction orthogonal to $<100>$ planes, referred to hereafter as $\Gamma-\mathrm{X}$ direction; (ii) direction orthogonal to $<110>$ planes, referred to hereafter as $\Gamma$-K direction and (iii) direction orthogonal to $<111>$ planes, referred to as $\Gamma$-L direction. Apart from the spatial orientation of the waveguide, there are several possibilities to drill a cylindrical hole in each of these facets stemming from the in-plane symmetrical properties. To keep the symmetry of the structure as high as possible we limited ourselves to only with few symmetrical points: two points for $\Gamma$-X $\mathrm{PCW}$, four points for $\Gamma-\mathrm{K}$ and four points for $\Gamma$-L (Fig. 3). Results in transmission differ significantly. This allows us to draw the 
conclusion of a strong dependence of transmission properties on the waveguide entry position. We identified positions providing the most effective performance of PCWs and fixed them for further simulations. In the following we present spectral dependencies of the FDTD calculated transmission through PCWs normalized to the transmission of the same layout of the source and the detectors, but in vacuum.
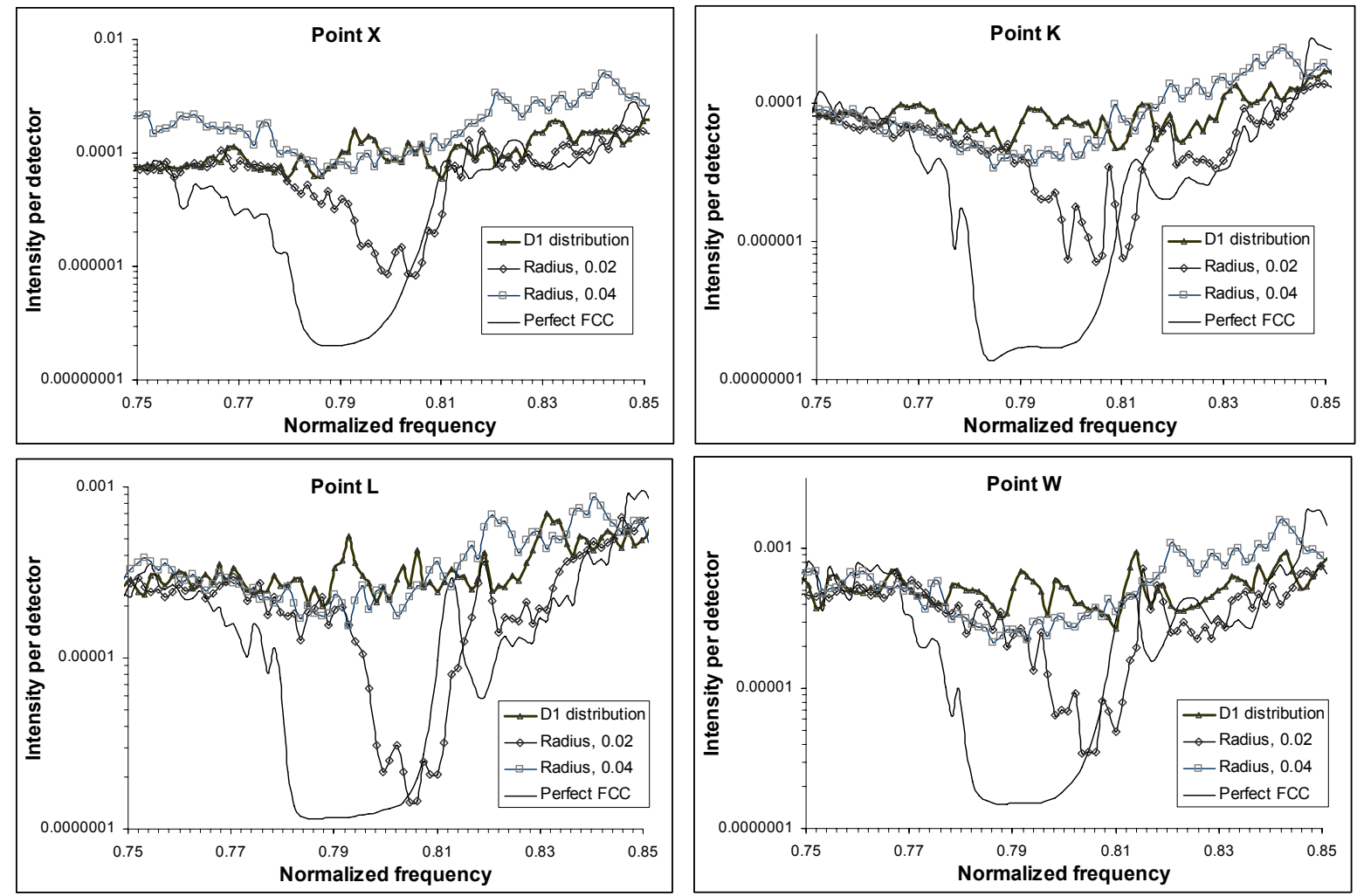

Figure 2. Direction dependent QDOS spectra for Si inverted opal with normal distribution of radius deviations and D1 distribution of radii (see the tables for specifications). Detection directions are shown in the charts titles. QDOS spectra for the perfect PhC in the same directions are shown by thin solid line.

Transmission spectra for the $\Gamma-\mathrm{K}$ directed $\mathrm{CoS}$ and $\mathrm{CPCW}$ waveguides of different radii are shown in Fig. 3 , left. From the point of view of the width of the transmission zone in addition to the transmission peak value, CoS with radius $r=0.48 a$ should be claimed to be the best. This result overrides the conclusion of [5], where CoS with $r=0.45 a$ was proclaimed as an example of single mode waveguide with the broadest possible range. However, in two other directions, $\Gamma$ - $\mathrm{L}$ and $\Gamma-\mathrm{X}, \mathrm{CoS}$ waveguides exhibit spiky behaviour and considerable drift in peak positions (Fig. 3, right). The level of normalized transmission is not high. In contrary, CPCW are more effective (Fig. 3, left). Therefore, we can conclude that $\mathrm{CoS}$ waveguides are promising for $\Gamma-\mathrm{K}$ direction only, while cylindrical waveguides of radius $r=0.40 a$ and larger might be quite universal.
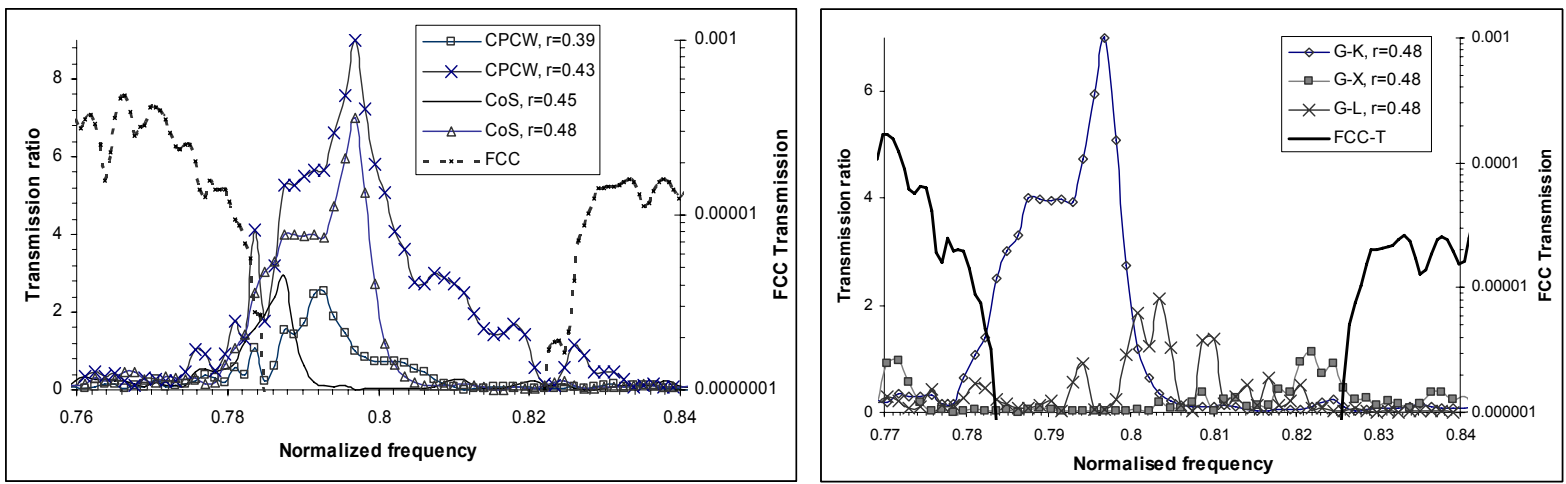

Figure 3. Left: Normalised transmission spectra for $\Gamma$ - K PCWs, thickness - 7a. Types of the waveguides and their radii are described in the table of curves specifications. Transmission spectrum for the perfect $P h C$ in $\Gamma-L$ is shown in black dashed line; Right: Normalised transmission spectra for CoS with $r=0.48$ a in $\Gamma$ - K, $\Gamma$ - X and $\Gamma$ - L directions. Transmission spectrum for perfect $P h C$ in $\Gamma$ - $L$ is shown in black solid line. 
To investigate the best overlap of effective transmission for all directions, transmission spectra are placed on the same charts. An example with the $\operatorname{CoS}(r=0.48 a)$ shows that there exists no overlapping zone with reasonable level of transmission (Fig.3, right). Moreover, positions of the peaks are at the different edges of PBG, so hardly the situation can be ameliorated even by means of intensive optimization. For radius $r=0.354 a$, example from [5], CPCWs have very poor effectiveness. By increasing the radius, transmission becomes much better (see Fig. 3, left). For example, one can find several zones, where transmission of $\mathrm{CPCW}$ is equal or higher 2.0 for all three directions, e.g. for $a / \lambda=0.794$.

Single-mode waveguides are preferable in photonic circuits. However, the tendency we observed for CPCW was that efficiency of guiding is higher for wider defects with radius $r=0.40 a$ and larger. With such radius CPW are believed to be multimode. One important investigation is the influence of polarization on the transmission spectra. Such data could provide evidence about multimode behaviour in the passbands. We performed calculation similar to those reported above, but changing initial polarization of the light source to the orthogonal one. Two main specifications were chosen, namely CPCW with $r=0.40 a$ and $\operatorname{CoS}$ with $r=0.45 a$. Transmission of orthogonally polarized light with the same entering point is nearly 3-4 times lower for CPCW, so it is possible to speculate if not on single-mode but at least on polarization sensitive behaviour of cylindrical waveguides.

\section{2PP INSCRIBING OF STRAIGHT AND BENT DEFECTS}

The first step towards the fabrication of defects involves the infiltration of the opal template, consisting of $1 \mu \mathrm{m}$ size polystyrene spheres, with the photo polymer ORMOCER ${ }^{\circledR}$. The infiltration is followed by the actual inscription of defects into the template through local solidification of the photo polymer by the 2PP. For this purpose we use a mode-locked frequency-doubled Ytterbium-doped glass laser which emits $240 \mathrm{fs}$ pulses at a wavelength of $515 \mathrm{~nm}$. The laser beam is focussed into the opal crystal by $20 \times$ and $100 \times$ microscope objectives, respectively. The laser focus can be moved to any position inside the sample by using xyz-translation stages and a scanner. After inscribing the vertical and horizontal defects into the $10-20 \mu \mathrm{m}$ thick opal films the remaining non-polymerized ORMOCER ${ }^{\circledR}$ is dissolved using isopropanol. Figure 4 shows some simple structures which can be combined to fabricate bent waveguides of any desired shape.
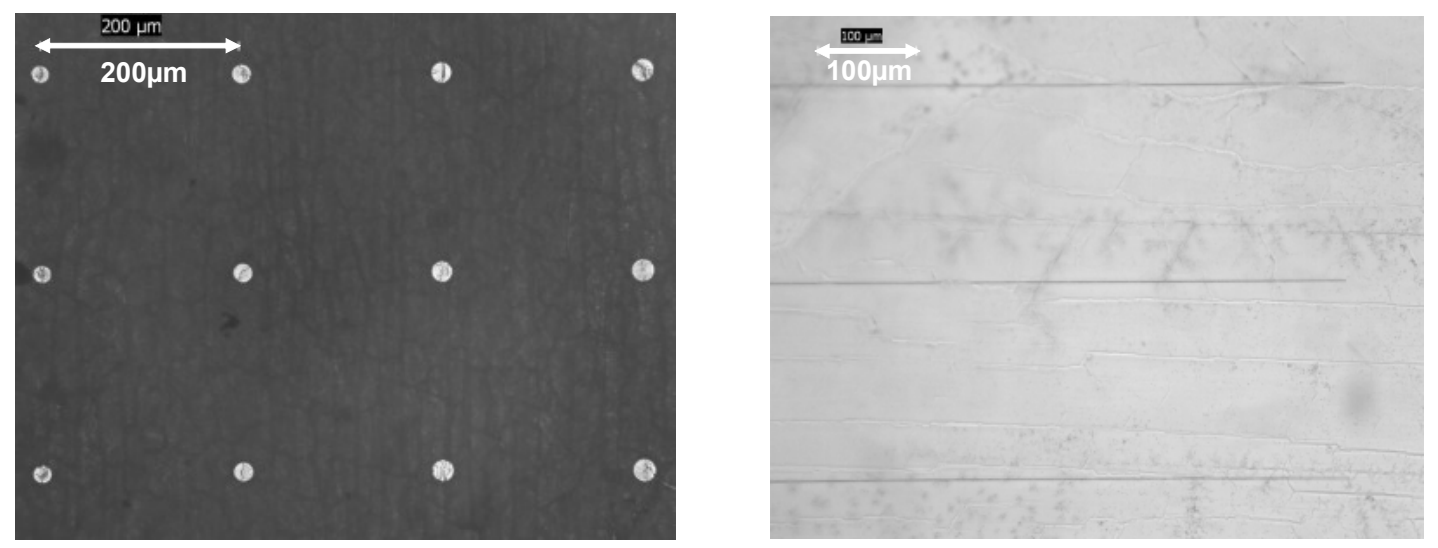

Figure 4. Optical microscope images of vertical waveguides with $20 \mu \mathrm{m}$ diameter (left) and horizontal waveguides with $1 \mu \mathrm{m}$ diameter (right) in polystyrene opals.

\section{CONCLUSIONS}

We have shown recent advances in designing towards all-optical integrated circuits on the basis of inverted opals. We demonstrated that various defects in $3 \mathrm{D}$ can be incorporated in the template with adequate quality too.

\section{REFERENCES}

[1] K. Takada, et al.: Improved spatial resolution and surface roughness in photopolymerization-based laser nanowriting, Appl. Phys. Lett., vol.86, pp. 1122-1124, 2005.

[2] S. A. Rinne, et al.: Embedded cavities and waveguides in three-dimensional silicon photonic crystals, Nature Photonics, vol.2, pp. 52-56, 2007.

[3] Z.-Y. Li, Z.-Q. Zhang, Fragility of photonic band gaps in inverse-opal photonic crystals, Phys. Rev. B, vol. 62, pp. 1516-1519, 2000.

[4] A. V. Lavrinenko, et. al.: Artificial Opals as Nanophotonic Materials for Optical Communications, in Proceedings of ICTON 2007, July 1-5, 2007, Rome, Italy, vol.2, p.208-211, 2007.

[5] V. Lousse, S. Fan, Waveguides in inverted opals photonic crystals, Opt. Express, vol. 14, pp. 866-878, 2006. 